\title{
Influência de estabilizantes na degradação foto-oxidativa de filmes de compósitos de SWy-1/poli(óxido de etileno)
}

\section{Influence of stabilizers on photo-oxidative degradation of SWy-1/poly (ethylene oxide) composite films}

\author{
Patrícia Coelho Lombardo ${ }^{1}$, Alessandra Lima Poli e Carla Cristina Schmitt²* \\ ${ }^{1}$ Departamento de Engenharia de Materiais, Escola de Engenharia de São Carlos, \\ Universidade de São Paulo - USP, São Carlos, SP, Brasil \\ ${ }^{2}$ Instituto de Química de São Carlos, Universidade de São Paulo - USP, São Carlos, SP, Brasil \\ *carla@iqsc.usp.br
}

\begin{abstract}
Resumo
Compósitos de poli(óxido de etileno) (PEO) com argila montmorilonita SWy-1 e estabilizantes (2-hidroxibenzofenona e tinuvin 770) foram preparados pelo método de intercalação em solução. Os filmes obtidos foram expostos a irradiação UV, e os produtos da fotodegradação foram monitorados por FTIR (Fourier Transform Infrared Spectroscopy, ou Espectroscopia no infravermelho por transformada de Fourier) e SEC (Size Exclusion Chromatography, ou cromatografia de exclusão por tamanho). O PEO puro apresentou maior coeficiente de degradação, $k_{d,}$ comparado com as demais amostras. O sistema que apresentou o menor valor para o coeficiente de degradação $\left(k_{d}=1,9 \times 10^{-6} \mathrm{~mol} \mathrm{~g}^{-1} \mathrm{~h}^{-1}\right)$ foi o compósito de $\mathrm{PEO} / 5 \% \mathrm{SWy}-1$ com $0,25 \%$ de tinuvin 770 . Nesse caso, a estabilização da matriz de $\mathrm{PEO}$ pode ser atribuída à argila juntamente com o tinuvin 770. A argila dispersa e absorve a irradiação UV, e o tinuvin age como estabilizante do tipo HALS (do inglês hindered amine light stabilizer, ou estabilizantes à luz tipo aminas impedidas).
\end{abstract}

Palavras-chave: poli(óxido de etileno), montmorilonita, fotoestabilizantes, degradação foto-oxidativa.

\begin{abstract}
Poly(ethylene oxide) (PEO) with SWy-1 montmorillonite and stabilizers (2-hydroxybenzophenone and tinuvin 770) composites were prepared by intercalation in solution method. The films of the composites were exposed to UV irradiation. The photodegradation products were monitored by FTIR (Fourier Transform Infrared Spectroscopy) and SEC (Size Exclusion Chromatography) method. The PEO pure showed higher degradation rate coefficients, $k_{d}$, compared to the others samples. The system that showed the lowest value for the degradation rate coefficients $\left(k_{d}=1.9 \times 10^{-6} \mathrm{~mol} \mathrm{~g}^{-1} \mathrm{~h}^{-1}\right)$ was the PEO/SWy-1 $5 \%$ composite with $0.25 \%$ of tinuvin 770 . In this case, the stabilization of the PEO matrix can be attributed to clay associated with tinuvin 770. The clay scatters and absorbs UV irradiation and tinuvin acts as a stabilizer of the HALS (hindered amine light stabilizers) kind.
\end{abstract}

Keywords: poly(ethylene oxide), montmorillonite, photostabilizers, photooxidative degradation.

\section{Introdução}

Materiais poliméricos podem sofrer degradação em diversos ambientes fazendo com que seu tempo de uso seja limitado. Os polímeros em geral quando expostos a radiação ultravioleta do sol desencadeiam uma série de reações químicas que juntamente com os efeitos fotofísicos, fotoquímicos da radiação solar, oxidativos do oxigênio atmosférico, hidrolíticos da água e de temperatura fazem com que ocorra a degradação do polímero ${ }^{[1,2]}$. A fotodegradação pode causar alterações no comprimento das cadeias do polímero, nas propriedades mecânicas, propriedades de barreira, mudança de coloração, surgimento de fissuras e fraturas, perda de brilho entre outras. Para tentar minimizar os efeitos degradativos causados pela longa exposição de materiais poliméricos à radiação solar, e assim aumentar o seu tempo de vida, aditivos fotoestabilizantes podem ser adicionados aos polímeros durante sua etapa de processamento ${ }^{[3]}$. Os efeitos da fotooxidação em polímero puro têm sido objeto de estudo por vários autores ${ }^{[4-6]}$.

Os danos causados pela foto-oxidação de materiais poliméricos também são aplicados aos materiais compósitos do tipo polímero/argila, no entanto, apesar de existir vários relatos na literatura a respeito de polímeros/silicatos, pouca atenção tem sido dada ao seu comportamento durante a fotodegradação ${ }^{[7-10]}$. 
Em geral, os estudos de processos foto-oxidativos de materiais compósitos polímero/argila mostram que a presença da argila acelera os processos de degradação dos mesmos quando comparados ao polímero puro ${ }^{[11-13]}$.

Morlat-Therias et al. ${ }^{[14]}$ estudaram a influência de dois tipos de aditivo antioxidante (fenólico e redox), durante a irradiação UV de nanocompósitos de polipropileno na presença de argila organofílica. Os autores observaram que os compósitos se foto-oxidam mais rapidamente em comparação com as outras amostras devido a presença de íons de ferro estrutural no argilomineral formando sítios catalíticos, a baixa eficiência do estabilizante ou a adsorção do estabilizante entre as lamelas de argila inibindo assim a sua ação.

Recentemente, Valandro et al. ${ }^{[7]}$ acompanharam por cromatografia de exclusão por tamanho (SEC) a influência da argila organofílica durante a fotodegradação de nanocompósitos de PMMA/SWy-1. Os resultados mostraram que a presença de argila minimizam os efeitos da irradiação UV.

Em nosso trabalho anterior foi abordada a influência da argila na fotodegradação de nanocompósitos de PEO/SWy-1. A argila inibe o processo de fotodegradação, atuando como estabilizador contra a radiação $\mathrm{UV}^{[8]}$.

Dessa maneira, no presente trabalho é investigado os efeitos da irradiação UV em filmes de compósitos de $\mathrm{PEO} / \mathrm{SWy}-1$ contendo dois tipos de fotoestabilizantes, 2-hidroxibenzofenona e tinuvin 770 .

\section{Experimental}

\subsection{Materiais e métodos}

A argila montmorilonita $\mathrm{SWy}-1 / \mathrm{Na}^{+}$utilizada na preparação dos compósitos foi adquirida da Source Clays, Clay Minerals Society, Universidade de Missouri, Columbia, MO. A argila foi purificada como descrito em trabalho anterior ${ }^{[15]}$. O poli(óxido de etileno) com massa molar de $100.000 \mathrm{~g} \mathrm{~mol}^{-1}$, os estabilizantes 2-hidroxibenzofenona (2-HB) $\left(198,22 \mathrm{~g} \mathrm{~mol}^{-1}\right)$ e tinuvin 770 (Tn 770) (480,72 $\mathrm{g} \mathrm{mol}^{-1}$ ) foram adquiridos da Sigma Aldrich.

\subsection{Preparação dos filmes}

Filmes de PEO puro, de compósitos PEO/SWy-1 e $\mathrm{PEO} / \mathrm{SWy}$-1/estabilizantes 2-HB e Tn 770 foram preparados através do método de intercalação em solução, utilizando acetonitrila (HPLC, Tedia) como solvente. Os filmes foram obtidos com $0,1 \%$ e $0,25 \%(\mathrm{~m} / \mathrm{m})$ de estabilizantes, $5 \%(\mathrm{~m} / \mathrm{m})$ de argila e $1 \%(\mathrm{~m} / \mathrm{v})$ de PEO. Suspensões de argila em acetonitrila permaneceram sob agitação constante por 24 horas, em seguida foram adicionadas solução de PEO e estabilizantes 2-HB e Tn 770. A mistura final permaneceu sob agitação constante até total evaporação do solvente, a fim de se obter o material seco.

O material seco foi então colocado numa prensa hidráulica entre camadas de papel alumínio por 1 minuto à $100{ }^{\circ} \mathrm{C} \mathrm{e} 14,5 \mathrm{kgf} / \mathrm{cm}^{2}$. Os filmes obtidos foram destacados do alumínio e armazenados.

\subsection{Irradiação dos filmes}

Os filmes foram irradiados em câmara de irradiação contínua (TECNAL TE-383), com 16 lâmpadas germicidas de UV (6 W cada) totalizando $96 \mathrm{~W}$, a $40{ }^{\circ} \mathrm{C}$.

A emissão das lâmpadas foi medida utilizando o espectrorradiômetro Luzchem SPR-01. A intensidade em $254 \mathrm{~nm}$ foi $134 \mathrm{~mW} / \mathrm{m}^{2}$ na mesma posição da amostra.

\subsection{Caracterização dos filmes}

Os filmes foram caracterizados por difração de raios X (DRX) em um difratômetro de raios X Rigaku Rotaflex modelo RU 200B (radiação de cobre, $\lambda=0,154 \mathrm{~nm}$ ) e por espectroscopia de infravermelho por transformada de Fourier em um equipamento Bomem-MB 100 com acessório de refletância total atenuada (ATR).

A formação dos produtos resultantes do processo da degradação UV foi acompanhada por FTIR e SEC.

A variação da massa molar ponderal média $\left(\overline{M_{w}}\right)$ foi acompanhada por SEC. Foram utilizadas três colunas em série (Shodex-OHPAK KB-806M). O fluxo utilizado foi de $1 \mathrm{~mL} \mathrm{~min}^{-1}$, temperatura de $35^{\circ} \mathrm{C}$ e água como fase móvel. A curva de calibração foi construída utilizando-se padrões de PEO (Varian Polymer Standards) com massa molar de 15.120 à $895.500 \mathrm{~g} \mathrm{~mol}^{-1}$. As amostras foram filtradas em celulose regenerada $(0,45 \mu \mathrm{m})$.

\section{Resultados e Discussões}

\subsection{Caracterização por difração de raios $X(D R X)$}

A técnica de difração de raios $X$ foi utilizada para verificar a distância interlamelar da argila SWy-1 pura e dos compósitos de PEO/SWy-1/estabilizantes Tn 770 e 2-HB. A distância interlamelar $\left(\mathrm{d}_{001}\right)$ foi calculada a partir da Equação de Bragg ${ }^{[16]}$.

A Figura 1 apresenta os difratogramas de raios $\mathrm{X}$ da argila SWy-1, do filme de PEO puro e dos filmes de PEO/SWy-1/ estabilizantes.

A argila $\mathrm{SWy}-1$ apresentou pico de difração em $2 \theta=7,4^{\circ}$ com distância interlamelar $\left(d_{001}\right)$ de aproximadamente $12 \AA$. No caso dos compósitos de $\mathrm{PEO} / \mathrm{SWy}-1$ na ausência e na presença dos estabilizantes pôde-se observar um deslocamento do pico para valores menores de $2 \theta\left(2 \theta \sim 5^{\circ}\right)$ que resulta em um aumento da distância interlamelar para valores de $18 \AA$.

De acordo com os resultados de DRX os compósitos obtidos foram do tipo intercalado, ou seja, o polímero se encontra inserido entre as galerias da argila.

Os valores da distância interlamelar aumentaram de $12 \AA$, no caso da amostra de argila, para valores próximos a $18 \AA$. No caso dos compósitos de PEO/SWy-1/ estabilizantes, a adição dos mesmos não influênciou na expansão da distância interlamelar da argila. Dessa maneira, conhecendo-se o valor da espessura da camada do silicato $(\sim 9,5 \AA)$, o aumento da distância interlamelar $\left(\Delta \mathrm{d}_{\mathrm{L}}\right)$ obtido é por volta de $8 \AA$, que está relacionado à espessura do polímero intercalado. Segundo Aranda e Ruiz-Hitzky ${ }^{[17]}$ quando se obtém valores de $\Delta \mathrm{d}_{\mathrm{L}}$ próximos de $8 \AA$ a conformação do polímero intercalado pode ser 
de duas maneiras: a primeira hipótese é a conformação zig-zag planar do polímero, nesse caso são necessárias duas camadas de polímero; já na segunda possibilidade o polímero estaria na sua forma helicoidal entre as camadas do silicato.

\subsection{Caracterização por FTIR}

Os espectros de FTIR dos filmes de compósitos de $\mathrm{PEO} / \mathrm{SWy}-1 /$ estabilizantes apresentaram as mesmas características quando comparados com o filme de
PEO/SWy-1 (Figura 2a). No trabalho anterior foi discutido em detalhes os espectros de FTIR de filmes de PEO com argila montmorilonita $\mathrm{SWy}-1^{[8]}$.

O espectro de FTIR da argila SWy-1 pura (Figura 2a) é composto por uma banda larga na região de 3000-3600 $\mathrm{cm}^{-1}$ devido ao estiramento da ligação O-H (hidroxila) e uma banda $1000-1200 \mathrm{~cm}^{-1}$ característica do estiramento da ligação Si-O-Si (siloxano) ${ }^{[18,19]}$. O PEO puro apresenta uma banda larga em $2881 \mathrm{~cm}^{-1}$ devido ao estiramento vibracional do grupo $-\mathrm{CH}\left(\right.$ do $\mathrm{CH}_{2}$ alifático

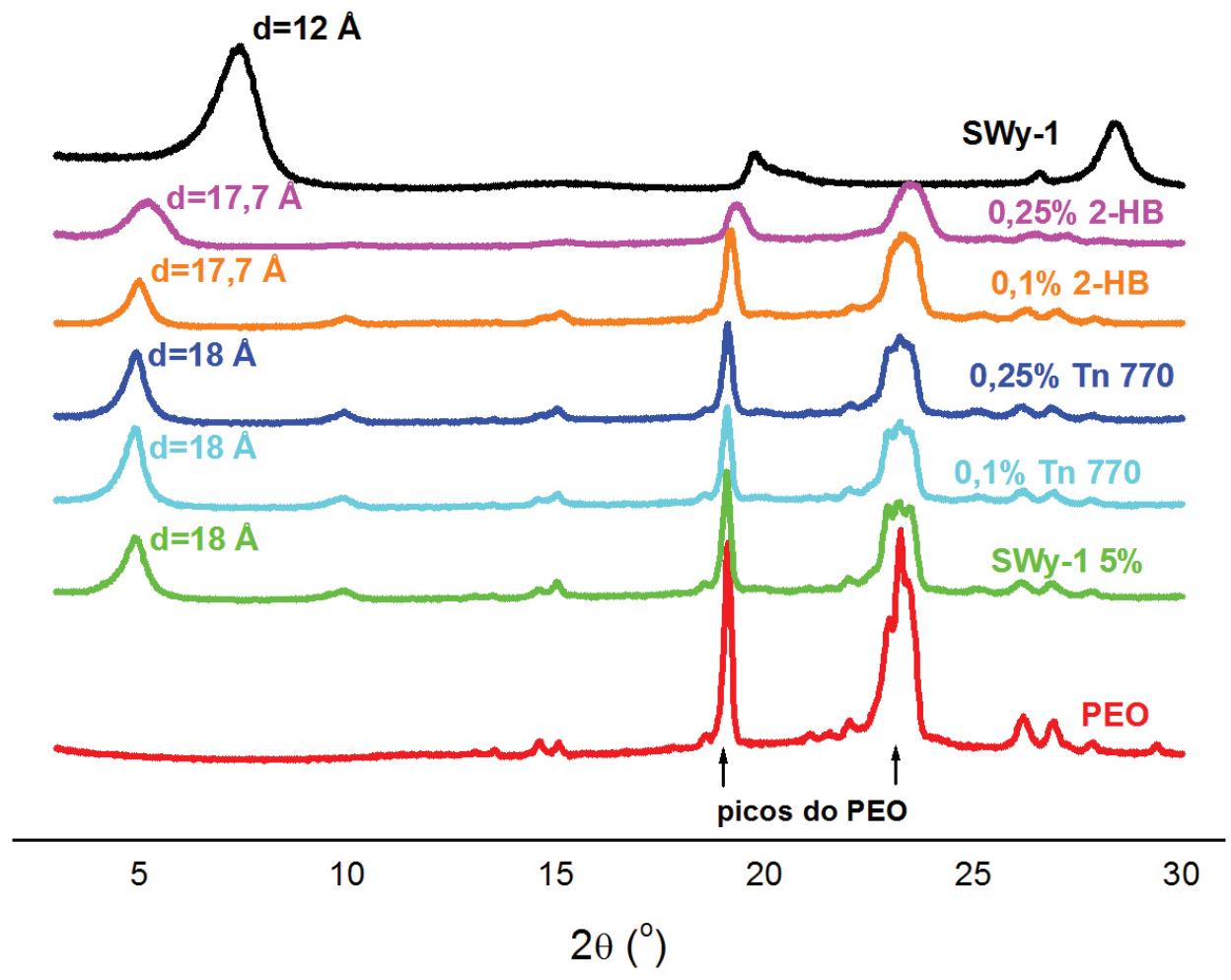

Figura 1. Difratogramas de raios X para a argila SWy-1 pura, PEO puro e compósitos de PEO/SWy-1/estabilizantes.

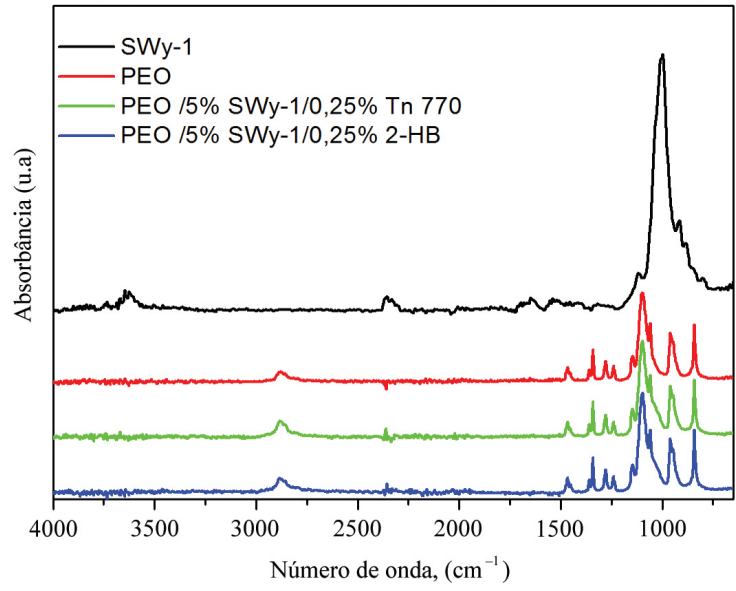

(a)

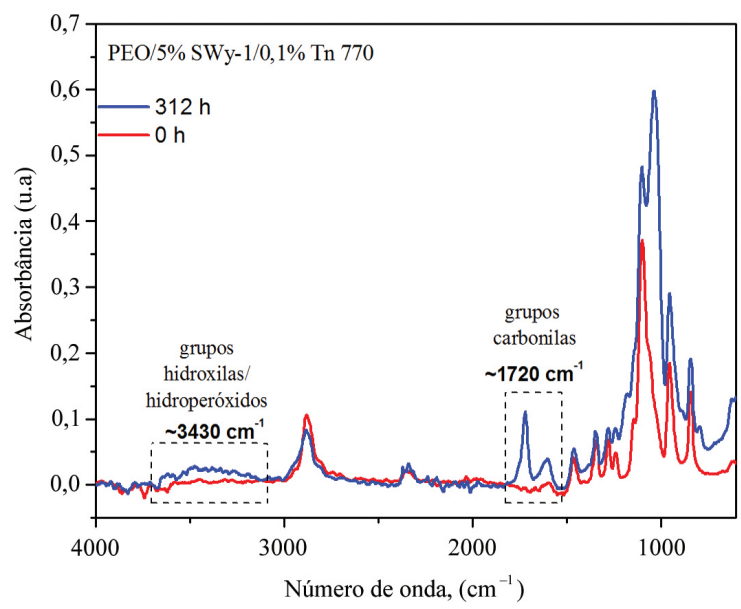

(b)

Figura 2. Espectros FTIR (a) dos filmes de PEO puro e compósitos de PEO/SWy-1 com 0,25\% Tn 770 e 0,25\% 2-HB; (b) filme de $\mathrm{PEO} / \mathrm{SWy}-1 /$ 0,1\% Tn 770 em função do tempo de irradiação. 
da unidade de óxido de etileno) ${ }^{[20,21]}$. As bandas em 1466, 1358,1342 e $957 \mathrm{~cm}^{-1}$ correspondem às vibrações de deformação do grupo C-H. Os picos na região de 1146 à $1061 \mathrm{~cm}^{-1}$ são atribuídos às vibrações de estiramento do grupo éter $(\mathrm{C}-\mathrm{O}-\mathrm{C})$ na fase cristalina ${ }^{[22]}$. A presença do pico em $957 \mathrm{~cm}^{-1}$ no espectro de FTIR, indica que o PEO se encontra em sua conformação helicoidal ${ }^{[23]}$.

\subsection{Degradação foto-oxidativa dos filmes com estabilizantes}

O comportamento de fotodegradação foi acompanhado por FTIR. A Figura $2 \mathrm{~b}$ apresenta as mudanças nos espectros de FTIR do filme do compósito PEO/5\% SWy-1 contendo $0,1 \%$ de tinuvin 770 antes e após irradiação UV.

Pode-se verificar que novas bandas são formadas em aproximadamente $1720 \mathrm{e} 3430 \mathrm{~cm}^{-1}$, resultantes da irradiação UV (Figura $2 b$ ). $\mathrm{O}$ aumento da banda em $1720 \mathrm{~cm}^{-1}$ pode ser atribuído à formação de espécies carbonila $(\mathrm{C}=\mathrm{O})^{[11,24]} \mathrm{e}$ a banda em $3430 \mathrm{~cm}^{-1}$ é atribuída a formação dos grupos hidroxilas e hidroperóxidos $(\mathrm{OH} / \mathrm{OOH})^{[14,19,25]}$. A formação destas espécies e o mecanismo geral proposto para o processo de degradação do $\mathrm{PEO}$ são descritos na literatura por Kaczmarek e colaboradores ${ }^{[22]}$.

\subsection{Cromatografia de exclusão por tamanho (SEC)}

Com a técnica de SEC foi possível acompanhar a variação da massa molar ponderal média $\left(\overline{M_{w}}\right)$ (Tabela 1$)$ bem como o número médio de quebra de cadeia por molécula (S) para as amostras de PEO puro e compósitos de PEO/SWy-1/estabilizantes.

O PEO puro e as amostras de PEO/2-HB $(0,1$ e $0,25 \%$ ) tiveram sua massa reduzida para $50 \%$ do seu valor inicial após $3 \mathrm{~h}$ de irradiação, a mesma redução só foi alcançada depois de $6 \mathrm{~h}$ para os compósitos de $\mathrm{PEO} / \mathrm{SWy}-1$ e PEO/SWy-1/2-HB independente da concentração do estabilizante 2-HB (Figura 3a).

Para todas as amostras com o estabilizante Tn 770, independente da concentração, tiveram sua massa reduzida para $50 \%$ do seu valor inicial após $3 \mathrm{~h}$ de irradiação (Figura 3b). A polidispersividade $\left(\overline{M_{w}} / \overline{M_{n}}\right)$ das amostras diminui ao longo da irradiação como mostra a Tabela 1.

O estreitamento das curvas cromatográficas para as amostras irradiadas indica que o polímero se torna mais uniforme em termos de distribuição de massa, o que significa que o polímero tende a ser menos disperso. Simultaneamente, um novo pico no tempo de retenção aparece nas curvas cromatográficas durante a irradiação (Figura 4), que pode ser atribuído à formação de produtos com menor massa molar. Resultados semelhantes foram obtidos por Morlat et al. ${ }^{[26]}$ para a oxidação de PEO em solução aquosa, onde os polímeros oxidados apresentaram menor polidispersividade que as amostras originais.

O progresso dos processos de degradação do $\mathrm{PEO}$ também foi avaliado pelo número médio de cisões de cadeia por molécula (S). Este parâmetro pode ser calculado de acordo com o modelo desenvolvido por Madras e $\operatorname{McCoy}{ }^{[27]}$ (Equação 1).

$$
S=\frac{\overline{M_{n}}(0)}{\overline{M_{n}}(t)}-1
$$

Pode-se observar que o número médio de quebra de cadeia por molécula (S) aumenta em função da irradiação, indicando que está ocorrendo a degradação do polímero em todas as amostras (Tabela 1).

Tabela 1. Valores da polidispersividade e número médio de quebra de cadeia por molécula (S) obtidos no tempo inicial e após a irradiação. Massa molar ponderal média inicial $\left(\overline{M_{w}}(0)\right)$ e massa molar numérica média inicial $\left(\overline{M_{n}}(0)\right)$. O coeficiente de degradação $\left(k_{d}\right)$ das amostras foi calculado a partir da Equação 2.

\begin{tabular}{|c|c|c|c|c|c|c|}
\hline & $t$ (h) & $\overline{M_{w}} / \overline{M_{n}}$ & $\mathbf{S}$ & ${\overline{M_{w}}}_{(\mathbf{0})}$ & $\overline{M_{n}}(0)$ & $\begin{array}{c}k_{d} \\
\left(10^{-6} \mathrm{~mol} \mathrm{~g}^{-1} \mathrm{~h}^{-1}\right)\end{array}$ \\
\hline \multirow{2}{*}{ PEO } & 0 & 3,4 & 0 & \multirow{2}{*}{67.355} & \multirow{2}{*}{20.108} & \multirow{2}{*}{5,5} \\
\hline & 144 & 1,2 & 5,2 & & & \\
\hline \multirow{2}{*}{$\mathrm{PEO} / 5 \% \mathrm{SWy}-1$} & 0 & 3,4 & 0 & \multirow{2}{*}{66.889} & \multirow{2}{*}{19.820} & \multirow{2}{*}{3,0} \\
\hline & 312 & 1,1 & 4,8 & & & \\
\hline \multirow{2}{*}{$\mathrm{PEO} / 0,1 \% 2-\mathrm{HB}$} & 0 & 3,2 & 0 & \multirow{2}{*}{80.609} & \multirow{2}{*}{24.870} & \multirow{2}{*}{8,9} \\
\hline & 144 & 1,1 & 7,1 & & & \\
\hline \multirow{2}{*}{ PEO $/ 0,25 \% 2-\mathrm{HB}$} & 0 & 3,8 & 0 & \multirow{2}{*}{81.379} & \multirow{2}{*}{21.175} & \multirow{2}{*}{8,5} \\
\hline & 144 & 1,2 & 6,0 & & & \\
\hline \multirow{2}{*}{$\mathrm{PEO} / 5 \% \mathrm{SWy}-1 / 0,1 \% 2-\mathrm{HB}$} & 0 & 3,4 & 0 & \multirow{2}{*}{67.889} & \multirow{2}{*}{19.708} & \multirow{2}{*}{4,0} \\
\hline & 312 & 1,1 & 5,2 & & & \\
\hline \multirow{2}{*}{ PEO $/ 5 \%$ SWy- $1 / 0,25 \%$ 2-HB } & 0 & 3,3 & 0 & \multirow{2}{*}{66.914} & \multirow{2}{*}{20.251} & \multirow{2}{*}{3,0} \\
\hline & 312 & 1,3 & 4,6 & & & \\
\hline \multirow{2}{*}{ PEO $/ 0,1 \%$ Tn 770} & 0 & 3,2 & 0 & \multirow{2}{*}{76.808} & \multirow{2}{*}{21.065} & \multirow{2}{*}{2,9} \\
\hline & 144 & 1,3 & 5,6 & & & \\
\hline \multirow{2}{*}{$\mathrm{PEO} /$ 0,25\% Tn 770} & 0 & 3,8 & 0 & \multirow{2}{*}{80.051} & \multirow{2}{*}{23.250} & \multirow{2}{*}{3,0} \\
\hline & 144 & 1,3 & 6,1 & & & \\
\hline \multirow{2}{*}{ PEO /5\% SWy-1/ 0,1\% Tn 770} & 0 & 3,4 & 0 & \multirow{2}{*}{78.598} & \multirow{2}{*}{21.062} & 29 \\
\hline & 312 & 1,1 & 5,5 & & & \\
\hline PFO $/ 5 \%$ SWV-1/ $025 \%$ Tn 770 & 0 & 3,3 & 0 & 73090 & 22140 & 10 \\
\hline 1 & 312 & 1,1 & 5,8 & ( & 22.147 & (1, \\
\hline
\end{tabular}




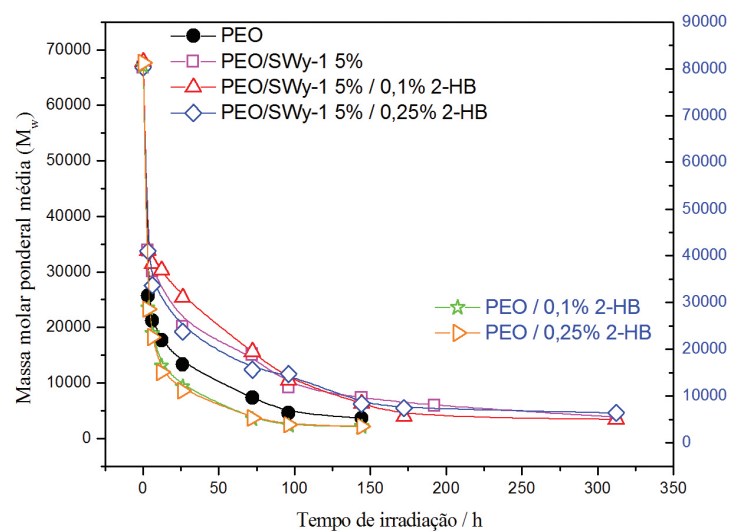

(a)

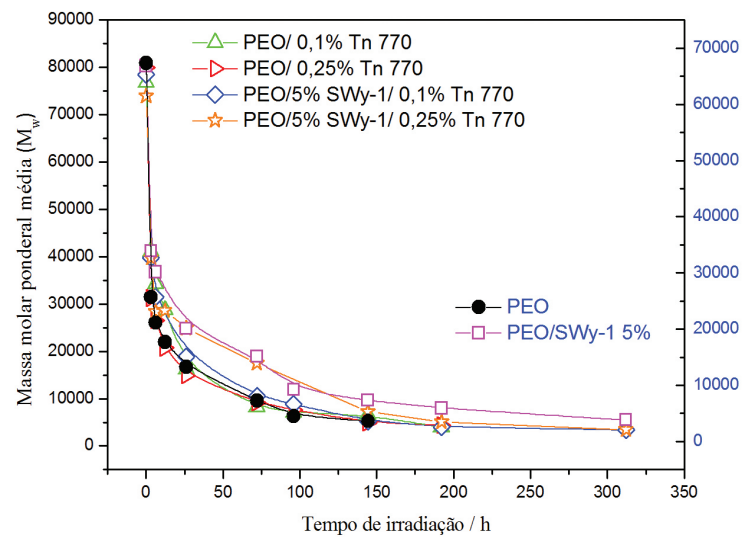

(b)

Figura 3. Massa molar ponderal média dos filmes de (a) PEO, PEO/2-HB, PEO/SWy-1 e PEO/SWy-1/2-HB e (b) PEO, PEO/Tn 770, $\mathrm{PEO} / \mathrm{SWy}-1$ e PEO/SWy-1/Tn 770.

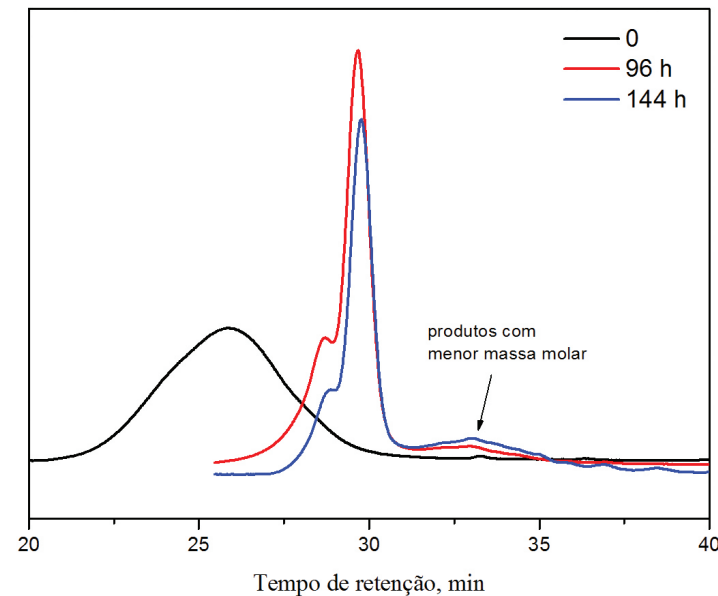

Figura 4. Cromatogramas de SEC do PEO puro antes e após a irradiação

O coeficiente de degradação $\left(k_{d}\right)$ foi calculado usando o modelo descrito por Marimuthu e Madras ${ }^{[28]}$ (Equação 2).

$$
\frac{\overline{M_{n}}(0)}{\overline{M_{n}}(t)}-1=\overline{M_{n}}(0) k_{d} t
$$

Essa relação está representada na Figura 5. Os coeficientes de degradação $\left(k_{d}\right)$ para o PEO puro e compósitos de PEO/SWy-1/estabilizantes foram calculados a partir dos primeiros pontos destas curvas (Figuras $5 \mathrm{~b}$ e $5 \mathrm{~d}$ ), utilizando os valores de $\left(\overline{M_{w}}\right)$ inicial para cada sistema. Os valores de $k_{d}$ estão apresentados na Tabela 1 .

De acordo com os resultados da Tabela 1, pode-se verificar que as amostras de $\mathrm{PEO} / 2-\mathrm{HB}$ apresentaram os maiores valores de $k_{d}$ de aproximadamente $9 \times 10^{-6} \mathrm{~mol}$ $\mathrm{g}^{-1} \mathrm{~h}^{-1}$, seguido pelo PEO com $k_{d}$ de $5,5 \times 10^{-6} \mathrm{~mol} \mathrm{~g}^{-1} \mathrm{~h}^{-1}$. As amostras de PEO/SWy-1, PEO/SWy-1/0,1\% 2-HB e $\mathrm{PEO} / \mathrm{SWy}-1 / 0,25 \%$ 2-HB apresentaram valores de $k_{d}$ de $3,0 \times 10^{-6}, 4,0 \times 10^{-6}$ e $3,0 \times 10^{-6} \mathrm{~mol} \mathrm{~g}^{-1} \mathrm{~h}^{-1}$, respectivamente. De acordo com os resultados pode-se dizer que no caso das amostras PEO/SWy-1/2-HB, a estabilização ocorreu devido à presença da argila e não do estabilizante 2-HB.
No caso do estabilizante Tn 770 é possível verificar que as amostras de PEO/Tn 770 apresentaram a mesma taxa de degradação que a amostra de $\mathrm{PEO} / \mathrm{SWy}-1\left(k_{d}=3,0 \times 10^{-6} \mathrm{~mol} \mathrm{~g}^{-1} \mathrm{~h}^{-1}\right)$. A amostras $\mathrm{PEO} / \mathrm{SWy}-1 / 0,25 \%$ Tn 770 apresentou menor coeficiente de degradação $\left(k_{d}=1,9 \times 10^{-6} \mathrm{~mol} . \mathrm{g}^{-1} \mathrm{~h}^{-1}\right)$ comparado ao PEO puro.

De maneira geral, os estudos de degradação fotooxidativa mostraram que o estabilizante 2 -HB não foi eficiente durante o processo fotodegradativo do PEO. De fato, Allen e colaboradores descrevem que o conceito de fotoestabilização em polímeros comerciais por absorção UV torna-se inviável do ponto de vista prático. Isso porque altas concentrações de um absorvedor seriam requeridas na superfície do sistema polimérico para uma operação eficiente, desde que as reações de foto-oxidação em polímeros ocorrem próximas a superfície ${ }^{[29]}$.

A molécula 2-hidroxibenzofenona é considerada a molécula base para a maioria dos estabilizantes absorvedores de UV. A molécula 2-hidroxibenzofenona e seus derivados apresentam em sua estrutura uma ligação intramolecular de hidrogênio envolvendo a hidroxila fenólica o qual está relacionado com a origem de sua ação estabilizante. A absorção da luz faz com que ocorra a formação de um estado excitado tripleto localizado na carbonila. No estado excitado pode ocorrer a reação de formação do "enol", no entanto, a forma "enol" é menos estável termodinamicamente que a forma cetona, ocorrendo a sua regeneração para o estado eletrônico fundamental com a liberação de energia na forma de calor ${ }^{[30]}$.

No caso do Tn 770 foi possível obter a mesma estabilização proporcionada pela argila $\mathrm{SWy}-1$ à matriz polimérica (PEO) com a adição de pequenas quantidades do mesmo. A adição do Tn 770 juntamente com a argila SWy-1 ao sistema PEO/SWy-1/0,25\% Tn 770 promoveu maior estabilidade ao compósito. Essa melhora pode ser atribuída a ação do Tn 770 juntamente com a estabilização proporcionada pela argila SWy-1.

A argila SWy-1 pode ser considerada como estabilizante contra irradiação UV ${ }^{[7,8]}$. O modo de estabilização da argila pode ser explicado devido à capacidade da argila não só 


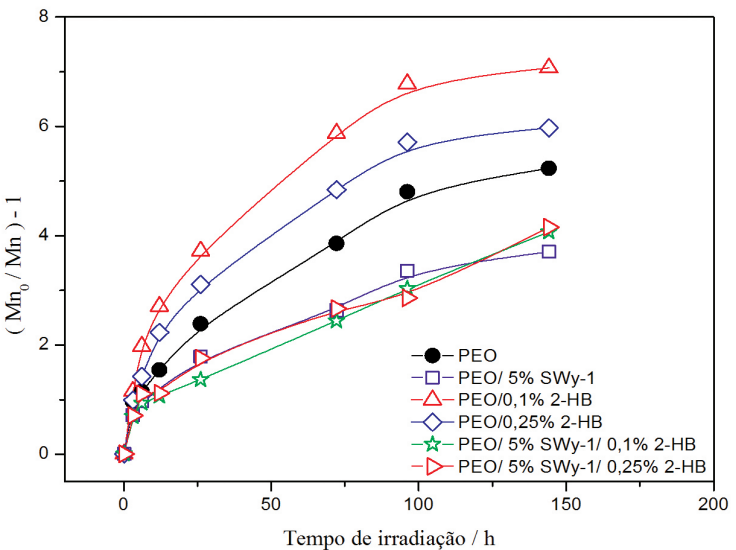

(a)

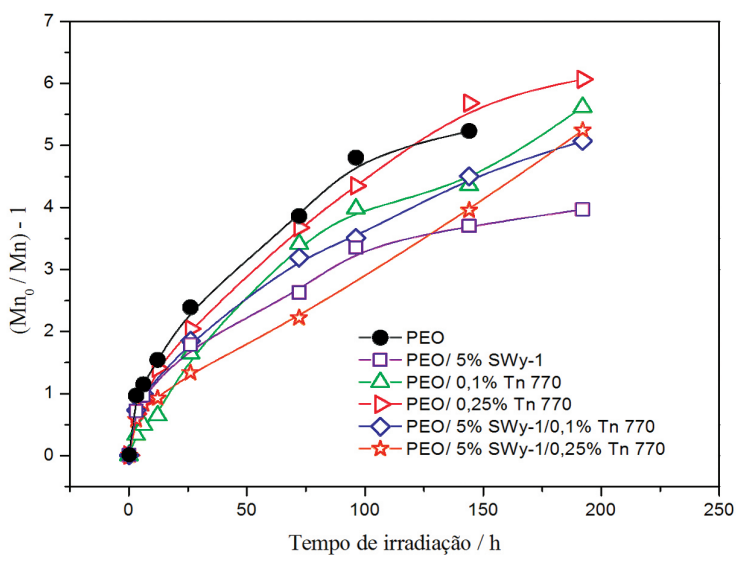

(c)

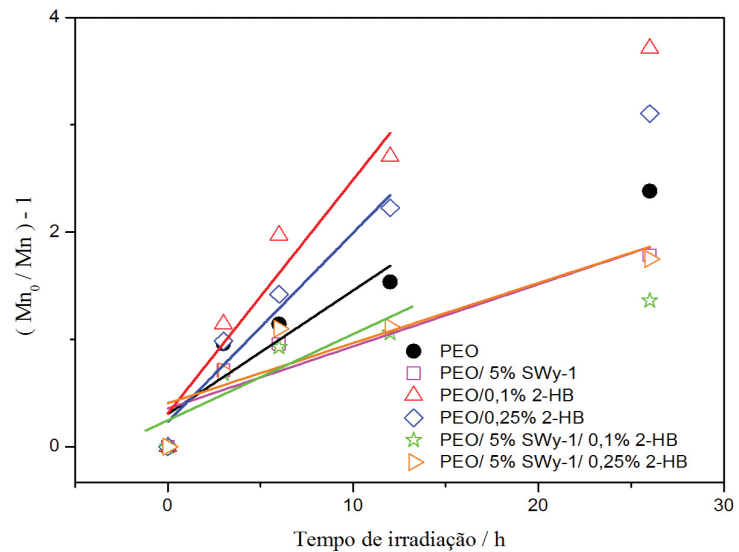

(b)

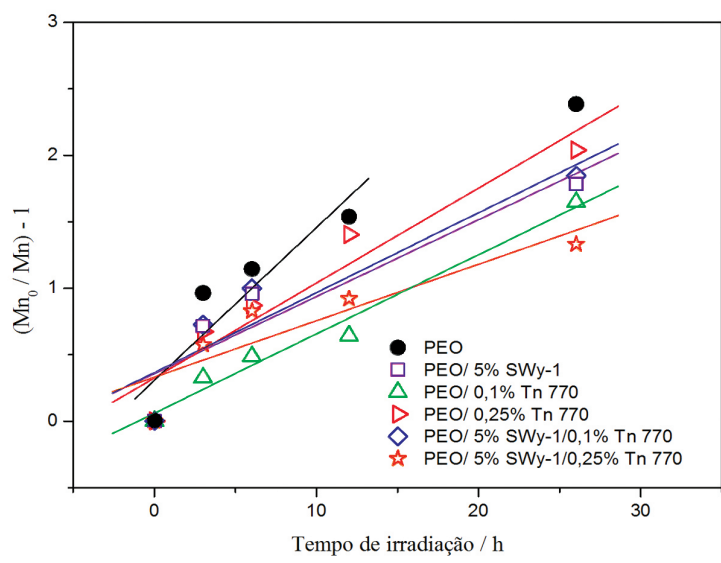

(d)

Figura 5. Gráfico de $\left[\overline{M_{n}}(0) / \overline{M_{n}}(t)\right]$ - 1 em função do tempo de irradiação (a) para os filmes de PEO, PEO/SWy-1 e PEO/SWy-1/HB e (c) filmes de PEO, PEO/SWy-1 e PEO/SWy-1/Tn. Os gráficos mostrados nas Figuras (b) e (d) destacam os tempos iniciais de irradiação, para cálculo do $k_{d}$. As retas nos gráficos (b) e (d) correspondem à regressão linear.

para espalhar a luz incidente, mas também para absorver parte da luz UV como discutido em artigo anterior ${ }^{[8]}$. O espectro de absorção da argila SWy-1 mostra banda em 242 nm, atribuída a uma transição de transferência de carga $\left(\mathrm{Fe}^{3+} \text { octaedral }\right)^{[31]}$. Assim, a absorção da argila minimiza a degradação de PEO. Essawy et al. ${ }^{[32]}$ observaram efeito semelhante para a fotodegradação dos compósitos de PVC/laponita, em que a adiação da laponita melhorou a resistência do polímero aos efeitos da irradiação UV.

Segundo Shi et al. ${ }^{[33]}$, o Tinuvin 770 é considerado um dos mais eficientes estabilizantes do tipo HALS devido à rápida conversão de amina em radicais nitroxilas.

Os fotoestabilizantes do tipo HALS, ou estabilizantes à luz tipo aminas impedidas, são aminas estericamente impedidas cuja ação estabilizante é ativada por uma reação fotoquímica. O mecanismo de estabilização é autorregenerativo, sua eficiência como estabilizante é devido a sua habilidade de formar radicais nitroxilas (NO.) estáveis, os quais são gerados pela oxidação da amina secundária $(=\mathrm{NH})$, que é um eficiente sequestrador de radicais alquila. Os radicais nitroxilas formados sequestram os radicais alquila do polímero (P.) produzidos durante a fotodegradação do polímero. Esses radicais nitroxilas estáveis podem sequestrar radicais alquila e radicais poliméricos alquila. A reação com radicais peroxilas faz com que ocorra a regeneração da nitroxila, fazendo com que o ciclo seja reiniciado ${ }^{[30,34]}$.

\section{Conclusão}

A metodologia utilizada para preparação dos filmes resultou em compósitos de $\mathrm{PEO} / \mathrm{SWy}-1 /$ estabilizantes do tipo intercalados.

Os estudos de degradação foto-oxidativa mostraram que o estabilizante 2-HB não foi eficiente durante o processo fotodegradativo do $\mathrm{PEO}$. O PEO puro degradou mais rápido em comparação com as amostras com estabilizante tinuvin 770. O tinuvin 770 proporcionou a mesma estabilidade que a argila montmorilonita $\mathrm{SWy}-1$ para a matriz polimérica de PEO. O sistema de $\mathrm{PEO} / 5 \% \mathrm{SWy}-1 \mathrm{com}$ $0,25 \%$ de Tn 770 apresentou o menor coeficiente de degradação $\left(k_{d}=1,9 \times 10^{-6} \mathrm{~mol} \mathrm{~g}^{-1} \mathrm{~h}^{-1}\right)$. Nesse último caso, a argila também contribui juntamente com o Tn 770 na estabilização da matriz de PEO minimizando assim os efeitos fotodegradativos. 


\section{Agradecimentos}

Os autores agradecem aos auxílios 2009/15998-1 e 2012-19656-0, Fundação de Amparo à Pesquisa do Estado de São Paulo (FAPESP) e ao CNPq e a Capes.

\section{Referências}

1. Ranby, B., \& Rabek, J. F. (1975). Photodegradation, photooxidation and photostabilization of polymers: principles and applications. London: John-Wiley.

2. Rabek, J. F. (1995). Polymer photodegradation. London: Chapman \& Hall. http://dx.doi.org/10.1007/978-94-011-1274-1.

3. De Paoli, M. A. (2008). Degradação e estabilização de polímeros. São Paulo: Artliber.

4. Santos, L. C., Poli, A. L., Cavalheiro, C. C. S., \& Neumann, M. G. (2009). The UV/H2O2 - photodegradation of poly(ethyleneglycol) and model compounds. Journal of the Brazilian Chemical Society, 20(8), 1467-1472. http://dx.doi. org/10.1590/S0103-50532009000800012.

5. Guadagno, L., Naddeo, C., \& Vittoria, V. (2004). Structural and Morphological Changes during UV Irradiation of the Crystalline Helical Form of Syndiotactic Polypropylene. Macromolecules, 37(26), 9826-9834. http://dx.doi. org/10.1021/ma040129j.

6. Morlat, S., \& Gardette, J. (2001). Phototransformation of water-soluble polymers. I: photo- and thermooxidation of poly(ethylene oxide) in solid state. Polymer, 42(14), 60716079. http://dx.doi.org/10.1016/S0032-3861(01)00084-2.

7. Valandro, S. R., Poli, A. L., Neumann, M. G., \& Schmitt, C. C. (2013). Organomontmorillonite/poly(methyl methacrylate) nanocomposites prepared by in situ photopolymerization. Effect of the organoclay on the photooxidative degradation. Journal of Applied Clay Science, 85, 19-24. http://dx.doi. org/10.1016/j.clay.2013.08.050.

8. Lombardo, P. C., Poli, A. L., Neumann, M. G., Machado, D. S., \& Schmitt, C. C. (2013). Photodegradation of poly(ethyleneoxide)/montmorillonite composite films. Journal of Applied Polymer Science, 127(5), 3687-3692. http://dx.doi. org/10.1002/app.37987.

9. Kumanayaka, T. O., Parthasarathy, R., \& Jollands, M. (2010). Accelerating effect of montmorillonite on oxidative degradation of polyethylene nanocomposites. Polymer Degradation \& Stability, 95(4), 672-676. http://dx.doi. org/10.1016/j.polymdegradstab.2009.11.036.

10. Oliveira, C. F. P., Demarquette, N. R., Carastan, D. J., \& Fechine, G. J. M. (2012). Fotodegradação de compósitos de poliestireno/argila montmorilonita: efeito do tipo de argila e presença de sal. Polímeros, 22(1), 13-21. http://dx.doi. org/10.1590/S0104-14282012005000010.

11. Qin, H., Zhang, Z., Feng, M., Gong, F., Zhang, S., \& Yang, M. J. (2004). The influence of interlayer cations on the photo-oxidative degradation of polyethylene/montmorillonite composites. Journal of Polymer Science. Part B, Polymer Physics, 42(16), 3006-3012. http://dx.doi.org/10.1002/ polb. 20068 .

12. Tidjani, A., \& Wilkie, C. A. (2001). Photo-oxidation of polymeric-inorganic nanocomposites: chemical, thermal stability and fire retardancy investigations. Polymer Degradation \& Stability, 74(1), 33-37. http://dx.doi. org/10.1016/S0141-3910(01)00061-1.

13. Qin, H., Zhang, S., Liu, H., Xie, S., Yang, M., \& Shen, D. (2005). Photo-oxidative degradation of polypropylene/ montmorillonite nanocomposites. Polymer, 46(9), 3149-3156. http://dx.doi.org/10.1016/j.polymer.2005.01.087.
14. Morlat-Therias, S., Mailhot, B., Gonzalez, D., \& Gardette, J. L. (2005). Photooxidation of Polypropylene/Montmorillonite Nanocomposites. 2. Interactions with Antioxidants. Chemistry of Materials, 17(5), 1072-1078. http://dx.doi.org/10.1021/ cm0401721.

15. Gessner, F., Schmitt, C. C., \& Neumann, M. G. (1994). TimeDependent Spectrophotometric Study of the Interaction of Basic Dyes with Clays. I. Methylene Blue and Neutral Red on Montmorillonite and Hectorite. Langmuir, 10(10), 3749-3753. http://dx.doi.org/10.1021/la00022a059.

16. Canevarolo, S. V., Jr. (2003). Técnicas de caracterização de polímeros. São Paulo: Artliber.

17. Aranda, P., \& Ruiz-Hitzky, E. (1992). Poly(ethylene oxide)silicate intercalation materials. Chemistry of Materials, 4(6), 1395-1403. http://dx.doi.org/10.1021/cm00024a048.

18. Kaczmarek, H., \& Podgórski, A. (2007). The effect of UV-irradiation on poly(vinyl alcohol) composites with montmorillonite. Journal of Photochemistry and Photobiology A Chemistry, 191(2-3), 209-215. http://dx.doi.org/10.1016/j. jphotochem.2007.04.025.

19. Morlat-Therias, S., Fanton, E., Gardette, J. L., Dintcheva, N. T., La Mantia, F. P., \& Malatesta, V. (2008). Photochemical stabilization of linear low-density polyethylene/clay nanocomposites: Towards durable nanocomposites. Polymer Degradation \& Stability, 93(10), 1776-1780. http://dx.doi. org/10.1016/j.polymdegradstab.2008.07.031.

20. Ratna, D., Abraham, T. N., \& Karger-Kocsis, J. (2008). Studies on polyethylene oxide and phenolic resin blends. Journal of Applied Polymer Science, 108(4), 2156-2162. http://dx.doi. org/10.1002/app.27883.

21. Volel, M., Armand, M., Gorecki, W., \& Saboungi, M.-L. (2005). Threading Polymer into Nanotubes: Evidence of Poly(ethylene oxide) Inclusion in Titanium Oxide. Chemistry of Materials, 17(8), 2028-2033. http://dx.doi.org/10.1021/ cm047880r.

22. Kaczmarek, H., Bajer, K., Gałka, P., \& Kotnowska, B. (2007). Photodegradation studies of novel biodegradable blends based on poly(ethylene oxide) and pectin. Polymer Degradation \& Stability, 92(11), 2058-2069. http://dx.doi.org/10.1016/j. polymdegradstab.2007.07.019.

23. Burgaz, E. (2011). Poly(ethylene-oxide)/clay/silica nanocomposites: Morphology and thermomechanical properties. Polymer, 52(22), 5118-5126. http://dx.doi. org/10.1016/j.polymer.2011.08.060.

24. Aranda, P., Mosqueda, Y., Pérez-Cappe, E., \& Ruiz-Hitzky, E. (2003). Electrical characterization of poly(ethylene oxide)clay nanocomposites prepared by microwave irradiation. Journal of Polymer Science. Part B, Polymer Physics, 41(24), 3249-3263. http://dx.doi.org/10.1002/polb.10704.

25. Neumann, M. G., Schmitt, C. C., \& Goi, B. E. J. (2010). Thioxanthone sensitized photodegradation of poly(alkyl methacrylate) films. Journal of Applied Polymer Science, 115(3), 1283-1288. http://dx.doi.org/10.1002/app.30758.

26. Morlat, S., \& Gardette, J. L. (2003). Phototransformation of water-soluble polymers. Part II: photooxidation of poly(ethylene oxide) in aqueous solution. Polymer, 44(26), 7891-7897. http://dx.doi.org/10.1016/j.polymer.2003.10.022.

27. Madras, G., \& Mccoy, B. J. (1997). Oxidative degradation kinetics of polystyrene in solution. Chemical Engineering Science, 52(16), 2707-2713. http://dx.doi.org/10.1016/S00092509(97)00091-2.

28. Marimuthu, A., \& Madras, G. (2007). Effect of Alkyl-Group Substituents on the Degradation of Poly(alkyl methacrylates) in Supercritical Fluids. Industrial \& Engineering Chemistry Research, 46(1), 15-21. http://dx.doi.org/10.1021/ie061068b. 
29. Allen, N. S., Edge, M., \& Chen, W. (1993). Thermal and photooxidative behaviour of 2-hydroxybenzophenone stabilisers in polyolefin films: Effect of 4-butoxy-4aminotetramethylpiperidine substitution. Polymer Degradation and Stability, 42(3), 293-306. http://dx.doi.org/10.1016/01413910(93)90225-8.

30. De Paoli, M. A. (2008). Degradação e estabilização de polímeros. São Paulo: Artliber.

31. Karickhoff, S. W., \& Bailey, G. W. (1973). Optical Absorption Spectra of Clay Minerals. Clays and Clay Minerals, 21(1), 59-70. http://dx.doi.org/10.1346/CCMN.1973.0210109.

32. Essawy, H. A., Abd El-Wahab, N. A., \& Abd El-Ghaffar, M. A. (2008). PVC-laponite nanocomposites: Enhanced resistance to UV radiation. Polymer Degradation \&
Stability, 93(8), 1472-1478. http://dx.doi.org/10.1016/j. polymdegradstab.2008.05.015.

33. Shi, W., Qu, B., \& Ranby, B. (1994). Photostabilization of photocrosslinked unsaturated polyesters. Polymer Degradation \& Stability, 44(2), 185-191. http://dx.doi. org/10.1016/0141-3910(94)90163-5.

34. Gijsman, P., Hennekens, J., \& Tummers, D. (1993). The mechanism of action of hindered amine light stabilizers. Polymer Degradation \& Stability, 39(2), 225-233. http:// dx.doi.org/10.1016/0141-3910(93)90099-5 .

Enviado: Nov. 12, 2013

Reenviado: Jun. 30, 2014 Aceito: Ago. 14, 2014 